\title{
Iterative ToA-Based Localization of Wireless Transmitters Using Dirichlet-Kernel-Based Range Representation
}

\author{
Evert I. Pocoma Copa*, François Rottenberg*†, François Quitin*, Luc Vandendorpe ${ }^{\dagger}$, \\ Philippe De Doncker*, François Horlin* \\ ${ }^{*}$ Université Libre de Bruxelles (ULB) \\ Av. Rooselvelt 50, 1050 Brussels, Belgium \\ Emails: \{epocomac,francois.rottenberg,fquitin,philippe.dedoncker,francois.horlin\}@ulb.be \\ †Université Catholique de Louvain (UCLouvain) \\ Place de l'Université 1, 1348 Ottignies-Louvain-la-Neuve, Belgium \\ Emails: \{francois.rottenberg,luc.vandendorpe\}@uclouvain.be
}

\begin{abstract}
Iterative localization is currently seen as an attractive solution to localize a transmitter in a cellular network. It has been shown that, by iterating between a range estimation step and a multi-lateration step, it is possible to refine the estimation in the first step, where only local information is used at iteration one. The iterative approach gets close to the performance of direct localization; nevertheless, it does not seem to converge to the direct localization performance for medium and low signal-to-noise-ratio values, due to the fact that it still suffers from loss of information due to projections and data representation. In this work, we propose to approximate the range log-likelihood at the base station with a Dirichlet kernel and to perform all the processing in a common $x y$-domain so that projections are no longer needed. We numerically show that our approach brings significant performance gains as compared to the time-of-arrival based iterative position estimation algorithm, getting really close to the performance of direct localization.

Index Terms-Localization, iterative processing
\end{abstract}

\section{INTRODUCTION}

Cellular networks have evolved towards increasingly accurate geo-location services. In 4G and 5G, localization has become an important functionality. What is more, a positioning reference signal (PRS) is included in the protocol to support device localization based on the estimation of the signal time-of-arrival (ToA) [1]. The direct position estimation (DPE) algorithm estimates the transmitter position using a grid search comparing the signals received by each base station (BS) to the signals that would theoretically be received if the transmitter was at that position [2], [3]. Therefore, all the baseband signals are communicated to a processing node known as fusion centre (FC), which demands high communication bandwidth between the BSs and the FC. To reduce the amount of communicated data a two-step localization algorithm can be used. It consists in a range estimation step at the BS followed by a multi-lateration step at the FC [4]. Some information is lost in the first step, since only local information is available at the BS. Moreover, [5] analytically demonstrates that the direct estimation approach DPE always outperforms the two-step approach.

For such a reason, the iterative position estimation (IPE) algorithm has been proposed in [6] that iterates over the two steps with the goal of refining the estimation in the first step. In the multi-lateration step, the IPE computes a different range prior distribution for each $\mathrm{BS}$, based on the range information computed in the first step at the BS and sent to the FC. Such range prior is then fed back to the BS to refine the range estimation in the first step. Moreover, the IPE algorithm introduces an iterative framework that has been extended and studied more in depth in [7] and [8] where the effect of multipath channels and time-misalignment between transmitter and receiver was studied over the algorithm. Finally, it has been also shown that the iterative framework proposed by the IPE algorithm could be used in different applications and scenarios such as angle of arrival (AOA)-based iterative localization [9] and localization on WLAN-like systems [10].

Nonetheless, the IPE algorithm does not seem to converge to the DPE performance due to the fact that it still suffers from two losses of information. First, since the BSs are located at different positions in the scene, the IPE algorithm projects the combined information of all other BSs from the $x y$-domain to the range domain of the particular BS to compute the prior information; hence, losing some information due to such projection. Second, it communicates the information between steps using the first two order moments of the range probabilistic density function (PDF), i.e., range mean and range variance. As explained in this paper, such parameters are not the best suited for the iterative framework specially for medium and low signal-to-noise-ratio (SNR) values, since the range distribution becomes a multi-modal distribution with only one of the modes/peaks giving information about the transmitter position and the remaining modes/peaks caused by 
noise. Therefore the selection of the correct mode can be done by taking advantage of the additional information sent by the other BSs from the second iteration on-wards.

Contributions: in this work two improvements of the IPE algorithm are proposed, so that its speed of convergence and mean square error (MSE) are enhanced. First, we propose to keep all the processing in the $x y$-domain to avoid any loss of information due to the projections onto the range domain. Second, we propose to use a Dirichlet kernel function to better approximate the range log-likelihood at the BS, using the iterative scheme to select the correct mode/peak of the range distribution based on the information shared by the other BSs.

The rest of the paper is organized as follows. Section II first introduces the orthogonal frequency-division multiplexing (OFDM) signal model. Section III secondly describes the proposed iterative positioning algorithm. After detailing mathematically all the approximations and assumptions a short summary of the new updated iterative framework is given. Section IV finally assesses numerically the performance in terms of MSE of the iterative algorithm and discusses the reasons for the performance improvement.

\section{SySTEM MODEL}

We consider a cellular network operating with the OFDM modulation. We assume a static transmitter that is simultaneously connected to $K$ time-synchronized BSs in its neighbourhood. Besides, we consider that the communication takes place in a communication bandwidth $B$. The OFDM modulation splits the communication bandwidth in orthogonal sub-carriers that are allocated to data or pilot symbols. A cyclic prefix $(\mathrm{CP})$ is added to each block of transmitted symbols to maintain the orthogonality between the sub-carriers even in the presence of channel time dispersion.

We consider that $P$ equispaced pilot sub-carriers are allocated along the communication bandwidth, i.e., the frequency difference between two consecutive pilot sub-carriers is constant and referred to as $\Delta_{f}$ measured in Hz. Only the pilot sub-carriers are considered in this work, but the study can be extended to a bigger group of considered sub-carriers as long as all of them are equispaced. For simplicity, the channel is considered to be a single propagation delay $\tau_{k}$ between the transmitter position $(x, y)$ and $\mathrm{BS}-k$. Additionally, the propagation delay is related to the distance $\delta_{k}$ between transmitter and BS- $k$ by the expression: $\tau_{k}=\delta_{k} / c$, where $c$ is the speed of light. If $\tau_{k}$ is shorter than the CP duration (which is a reasonable assumption for typical system parameters), the signal received on the pilot $\mathrm{p}$ at $\mathrm{BS}-k$ is

$$
r_{k p}=s_{p} e^{-j \frac{2 \pi p \Delta_{f}}{c} \delta_{k}}+w_{k p},
$$

where $r_{k, p}$ and $w_{k, p}$ are the received signal and corrupting noise respectively, for pilot sub-carrier- $p$ at BS- $k$, and $s_{p}$ is the symbol at pilot sub-carrier- $p$. The noise $w_{k p}$ is assumed to be independent zero mean circularly symmetric complex Gaussian of variance $\sigma_{w}^{2}$ and equal in all BSs.
Finally, similarly to the system model adopted in [6], a vector model is constructed at each $\mathrm{BS}-k$ by stacking all the received pilot symbols as

$$
\mathbf{r}_{k}=\mathbf{s}\left(\delta_{k}\right)+\mathbf{w}_{k}
$$

with

$$
\begin{gathered}
\mathbf{r}_{k}=\left[r_{k, 1} \ldots r_{k, P}\right]^{T} \\
\mathbf{w}_{k}=\left[w_{k, 1} \ldots w_{k, P}\right]^{T}, \\
\mathbf{s}\left(\delta_{k}\right)=\left[s_{1} e^{-j 1 \varphi \delta_{k}} \ldots s_{P} e^{-j P \varphi \delta_{k}}\right]^{T},
\end{gathered}
$$

where the constant $\varphi$ is used to simplify the notation and it is defined as $\varphi=\frac{2 \pi \Delta_{f}}{c}$.

\section{Iterative Positioning Algorithm}

The DPE algorithm estimates the transmitter position based on all the received signals; thus, all the received baseband signals collected by the BSs must be communicated and gathered in a FC. The posterior distribution of the transmitter position is given by (6), where $\alpha$ is a normalization factor ensuring that the integral of the posterior distribution in the scene is 1 , and $p(x, y)$ is the prior PDF of the transmitter position

$$
p\left(x, y \mid \mathbf{r}_{1}, \ldots \mathbf{r}_{K}\right)=\frac{1}{\alpha} \prod_{k=1}^{K} p_{k}\left(\mathbf{r}_{k} \mid x, y\right) p(x, y) .
$$

We assume that the transmitter can arbitrarily be located on a finite rectangle in the scene; hence, $x$ and $y$ are two mutually independent uniformly distributed random variables. Therefore, the prior PDF is expressed as $p(x, y)=p(x) p(y)$, where $p(x)$ is uniformly distributed for $x \in\left[x_{\min }, x_{\max }\right]$ and 0 elsewhere; and $p(y)$ is also uniformly distributed for $y \in$ $\left[y_{\min }, y_{\max }\right]$ and 0 elsewhere. Further, taking the logarithm of (6) yields the log-posterior distribution written as

$$
\mathcal{P}(x, y)=\sum_{k=1}^{K} \mathcal{L}_{k}\left(\mathbf{r}_{k} \mid x, y\right)+b,
$$

where $\mathcal{P}(x, y)$ denotes the log-posterior distribution of the transmitter position, i.e., $\mathcal{P}(x, y)=\log \left(p\left(x, y \mid \mathbf{r}_{1}, \ldots \mathbf{r}_{K}\right)\right)$. The first term on the right-hand side of (7) is nothing else than the sum of the log-likelihoods of the received signal at each $\mathrm{BS}$, i.e., $\mathcal{L}_{k}\left(\mathbf{r}_{k} \mid x, y\right)=\log \left(p_{k}\left(\mathbf{r}_{k} \mid x, y\right)\right)$; and $b$ is a constant term considering the normalisation factor and the uniformly distributed prior.

In order not to communicate the complete received signal $\mathbf{r}_{k}$ to the FC or to any other node, we look for a function that depends on just a few parameters, to represent the log-likelihood as accurately as possible. Based on (2), the log-likelihood of the received signal at BS- $k$ can be expressed as in (8), where $\delta_{k}$ is the range between transmitter and BS- $k$; hence, $\mathbf{s}\left(\delta_{k}\right)$ is defined for all values in the $x y$-domain search grid, i.e., $\delta_{k}(x, y)$. 


$$
\mathcal{L}_{k}\left(\mathbf{r}_{k} \mid x, y\right)=-\frac{1}{\sigma_{w}^{2}}\left(\mathbf{r}_{k}-\mathbf{s}\left(\delta_{k}\right)\right)^{H}\left(\mathbf{r}_{k}-\mathbf{s}\left(\delta_{k}\right)\right)-P \log \left(\pi \sigma_{w}^{2}\right)
$$

Further, exactly equal to the approach proposed in [6], the expression can be simplified as shown in (9), where all the constant terms, such as the received power $\mathbf{r}_{k}^{H} \mathbf{r}_{k}$ and transmitted power $\mathbf{s}\left(\delta_{k}\right)^{H} \mathbf{s}\left(\delta_{k}\right)$, have been gathered in a common coefficient $b_{k}$. Lastly, $\Re($.$) denotes the real part$ operator.

$$
\mathcal{L}_{k}\left(\mathbf{r}_{k} \mid x, y\right)=\frac{2}{\sigma_{w}^{2}} \Re\left(\mathbf{r}_{k}^{H} \mathbf{s}\left(\delta_{k}\right)\right)+b_{k}
$$

Notice that the received signal at BS- $k$ is a single vector defined as $\quad \mathbf{r}_{k}=\mathbf{s}\left(\tilde{\delta}_{k}\right)+\mathbf{w}_{k}$, where $\tilde{\delta}_{k}$ is the actual range to be estimated between transmitter and BS- $k$. Therefore, we can rewrite (9) as

$$
\mathcal{L}_{k}\left(\mathbf{r}_{k} \mid x, y\right)=\frac{2}{\sigma_{w}^{2}} \Re\left(\mathbf{s}\left(\tilde{\delta}_{k}\right)^{H} \mathbf{s}\left(\delta_{k}\right)\right)+\frac{2}{\sigma_{w}^{2}} \Re\left(\mathbf{w}_{k}^{H} \mathbf{s}\left(\delta_{k}\right)\right)+b_{k},
$$

By inspecting (10), we can make two remarks. First, we consider that the noise term $\mathbf{w}_{k}$ is uncorrelated with the transmitted signal $\mathbf{s}\left(\delta_{\mathbf{k}}\right)$; hence, at high SNR values, the second term in the right hand side of (10) will be smaller than the first one. While for medium and low SNR values the two terms can be comparable, as a result the range PDF becomes a multi-modal distribution but only one of the modes/peaks gives information about the transmitter position. Therefore, if the correct range mode $\tilde{\delta}_{k}$ is chosen, the other modes caused by noise can be omitted. Finally, we propose to omit the noise term, even though it could lead to the selection of a wrong range mode, and further we propose to use the iterative scheme to correct the selection of the correct range mode $\tilde{\delta}_{k}$.

Second, the first term can be rewritten as a sum of cosines by developing the real part operator. The two remarks are applied to (10) and the new expression is

$$
\mathcal{L}_{k}\left(\mathbf{r}_{k} \mid x, y\right) \approx \frac{2}{\sigma_{w}^{2}} \sum_{p=1}^{P}\left|s_{p}\right|^{2} \cos \left(p \varphi\left(\delta_{k}-\tilde{\delta}_{k}\right)\right)+b_{k} .
$$

Note that the Dirichlet kernel is also defined as a sum of cosines as shown in (12), where $P$ is the order of the Dirichlet kernel and the coefficient $1 /(1+2 P)$ ensures a maximum amplitude to be equal to one.

$$
\mathcal{D}_{P}(u) \triangleq \frac{1}{1+2 P}\left(1+2 \sum_{p=1}^{P} \cos (p u)\right)
$$

Therefore, we can rewrite (11) in terms of a Dirichlet kernel, as shown in (13), where we considered (as it is common) equipowered pilots, i.e., $\left|s_{p}\right|=1$. Notice that the constant term $-1 / \sigma_{w}^{2}$ is added as a result of replacing the sum of cosines by the Dirichlet kernel in (11).

$$
\mathcal{L}_{k}\left(\mathbf{r}_{k} \mid x, y\right) \approx \frac{1+2 P}{\sigma_{w}^{2}} \mathcal{D}_{P}\left(\varphi\left(\delta_{k}(x, y)-\tilde{\delta}_{k}\right)\right)+b_{k}-\frac{1}{\sigma_{w}^{2}}
$$

Finally, we approximate the $\log$-likelihood $\mathcal{L}_{k}\left(\mathbf{r}_{k} \mid x, y\right)$ by a function $\mathcal{M}_{k}(x, y)$, i.e.

$$
\mathcal{L}_{k}\left(\mathbf{r}_{k} \mid x, y\right)=\mathcal{M}_{k}(x, y)+\mathcal{E}_{k}(x, y),
$$

where $\mathcal{E}_{k}(x, y)$ is the error for such approximation. Based on (13) we define $\mathcal{M}_{k}(x, y)$ as a Dirichlet kernel of order $P$ centered at range $\tilde{\delta}_{k}$ and amplitude $a_{k}$ as

$$
\mathcal{M}_{k}(x, y)=a_{k} \mathcal{D}_{p}\left(\varphi\left(\delta_{k}(x, y)-\tilde{\delta}_{k}\right)\right),
$$

The function $\mathcal{M}_{k}$ is completely defined with the two parameters $\tilde{\delta}_{k}$ and $a_{k}$. Notice that we choose to define the amplitude $a_{k}$ as

$$
a_{k}=\left.\mathcal{L}_{k}\left(\mathbf{r}_{k} \mid x, y\right)\right|_{x=\tilde{x}_{k}, y=\tilde{y}_{k}},
$$

where $\tilde{x}_{k}, \tilde{y}_{k}$ is the location of the maximum peak of the approximated log-posterior defined in (17); and not directly equal to $(1+2 P) / \sigma_{w}^{2}$, to take into account the small effect of the discarded term in (10) over the magnitude of the peak of the Dirichlet kernel. Lastly, all the constant terms in (13) can be included/handled in the normalization of the distribution, and thus they are not taken into account in (15). The main reason for approximating the range log-likelihood defined in (8) with (14) is to simplify the exchange of information among the BSs.

Distributing the FC to the nodes; therefore, assuming a fully connected network, each BS- $k$ builds an approximation of the log-posterior denoted as $\hat{\mathcal{P}}_{k}$ as shown in (17), where the communicated parameters by all other BSs are used to approximate each of their log-likelihoods. Notice that the constant term is omitted since it can be handled in the normalization.

$$
\hat{\mathcal{P}}_{k}(x, y)=\mathcal{L}_{k}\left(\mathbf{r}_{k} \mid x, y\right)+\sum_{j \neq k}^{K} \mathcal{M}_{j}(x, y)
$$

As done in the original IPE algorithm proposed in [6], the estimated transmitter position $(\hat{x}, \hat{y})$ can be computed in the minimum mean squared error (MMSE) sense by computing the expectation of the posterior distribution as shown in (18). Notice that the expectation is regarding the approximated posterior distribution $\hat{p}_{k}=\frac{1}{\alpha} \exp \left(\hat{\mathcal{P}}_{k}\right)$, where the denominator $\alpha$ is nothing else than a normalization factor ensuring that the integral of $\hat{p}_{k}$ over the scene is 1 .

$$
\hat{x}=\mathbb{E}\left[x \mid \mathbf{r}_{1}, \ldots \mathbf{r}_{K}\right] \quad \hat{y}=\mathbb{E}\left[y \mid \mathbf{r}_{1}, \ldots \mathbf{r}_{K}\right]
$$

In summary, the iterative procedure proposed in [6] can be updated and outlined in the two following steps.

\section{A. Range Estimation / Refinement:}

This step takes place at each BS- $k$, and it has the goal of estimate the parameters of the function $\mathcal{M}_{k}(x, y)$, such that the approximated range log-likelihood is communicated to other BSs with only two parameters. It estimates the parameters $a_{k}, \tilde{\delta}_{k}$ based on the knowledge of the approximated 
posterior $\hat{\mathcal{P}}_{k}(x, y)$ defined in (17) of the previous iteration. The parameter $\tilde{\delta}_{k}$ is computed as the range between the BS- $k$ position and the maximum point of the approximated posterior, i.e.

$$
\begin{gathered}
\tilde{x}_{k}, \tilde{y}_{k}=\arg \max _{x, y}\left(\hat{\mathcal{P}}_{k}(x, y)\right), \\
\tilde{\delta}_{k}=\sqrt{\left(\tilde{x}_{k}-x_{b s_{k}}\right)^{2}+\left(\tilde{y}_{k}-y_{b s_{k}}\right)^{2}},
\end{gathered}
$$

where $\left(x_{b s_{k}}, y_{b s_{k}}\right)$ is the position of BS- $k$. At iteration 1, all $\mathcal{M}_{j \neq k}$ are zero, and only the local information is used in (19), i.e., $\hat{\mathcal{P}}_{k}(x, y)=\mathcal{L}_{k}\left(\mathbf{r}_{k} \mid x, y\right)$. What is more, at first iteration there are multiple tuples $\left(\tilde{x}_{k}, \tilde{y}_{k}\right)$ that are the maximum points of the $x y$-domain log-posterior at BS- $k$, but all of them are at the same range $\tilde{\delta}_{k}$.

Notice that $\left(\tilde{x}_{k}, \tilde{y}_{k}\right)$ are different from the estimated transmitter position $(\hat{x}, \hat{y})$, since $\left(\tilde{x}_{k}, \tilde{y}_{k}\right)$ are related to the selected mode of the multi-modal range PDF, while $(\hat{x}, \hat{y})$ are computed in (18).

\section{B. Range Prior Communication / Reconstruction}

In this step, BS $k$ sends the parameters $a_{k}, \tilde{\delta}_{k}$ computed in the previous step; and receives the parameters $a_{j}, \tilde{\delta}_{j}$ from all other BSs. Then it reconstructs each range log-likelihood approximation $\mathcal{M}_{j}(x, y)$ using (15) and updates the approximated posterior $\hat{\mathcal{P}}_{k}(x, y)$ using (17) to finally go back to Estimation/Refinement step. Notice that the $\mathcal{M}_{j}(x, y)$ is defined and reconstructed in the $x y$-domain. What is more, the log-likelihood $\mathcal{L}_{k}=\left(\mathbf{r}_{k} \mid x, y\right)$ is also in the same domain, hence there is no need of any projections since all the terms in (17) are compatible.

Notice that in the first iteration, the parameters $a_{k}, \tilde{\delta}_{k}$ are computed based on the local information only; hence, at medium and low SNR the selected $\tilde{\delta}_{k}$ might not be the right mode of the multi-modal range distribution. Nevertheless, thanks to the information shared by the other BS, the selection of the right mode $\tilde{\delta}_{k}$ can be corrected at the second iteration.

\section{Numerical Results}

The performance of the proposed algorithm is investigated by assessing the MSE using Monte-Carlo simulations. The considered scene consists on $K=4$ BSs located on the corners of a $100 \mathrm{~m}$-sided square. The transmitter lies at arbitrary positions inside the rectangular scene and communicates with the BSs over a bandwidth of $20 \mathrm{MHz}$. At each BS, the processing is done using a single OFDM symbol containing $P=64$ equispaced pilots with $\Delta_{f}=312.5 \mathrm{kHz}$. The MSE of the estimator is averaged over 1000 transmitter positions, channel and noise realizations. The SNR is defined as $S N R=\sum_{p}\left|s_{p}\right|^{2} /\left(P \sigma_{w}^{2}\right)$.

All the BSs have similar MSE curves when using the proposed algorithm; hence, only the MSE of BS one is shown in Figure 1. Further, the MSE is compared to the centralized DPE algorithm used as a reference, as in [6]. It can be seen that the MSE of the proposed algorithm gets very close to the one of the centralized DPE algorithm.

We also show in Figure 2 the MSE of the IPE algorithm proposed in [6], where the $\mathrm{FC}$ has been divided and distributed

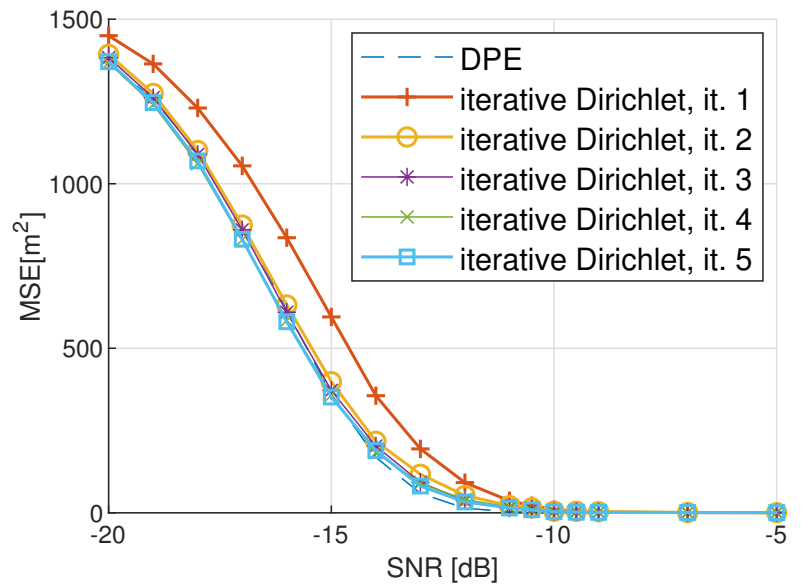

Fig. 1: MSE of transmitter position for the proposed algorithm

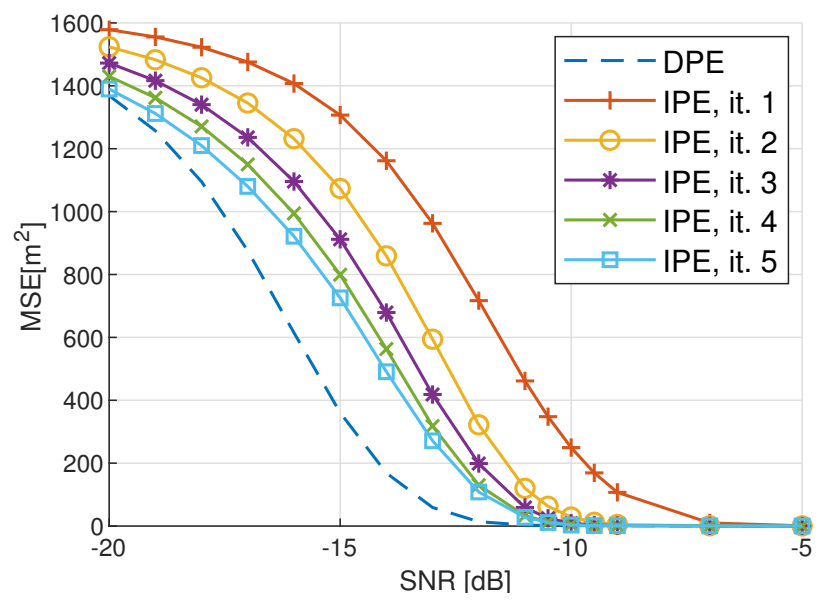

Fig. 2: MSE of transmitter position for the IPE algorithm proposed in [6]

to the BSs, so that each BS can compute the transmitter position. It can be clearly seen that: 1) our algorithm converges faster than the IPE (about two iterations) and 2) our algorithm closely converges to the DPE performance while the IPE does not.

As mentioned before, such improvement comes from the chosen model used to communicate the range log-likelihood combined with the iterative scheme to select the correct mode of the multi-modal range distribution at medium and low SNR values. To better show such advantage, a single realization is analyzed for different SNR values, where the transmitter was located at a range $\tilde{\delta}=81.1 \mathrm{~m}$ from the BS. Figures $3 \mathrm{a}$ and $4 \mathrm{a}$ show the range log-likelihood defined in (9) omitting the constant term $b_{k}$. Notice that, for the sake of visual comparison, the peak value of (9) was added as a constant to the range log-likelihood that uses the range mean and variance, such that all range log-likelihoods have the same maximum peak. 


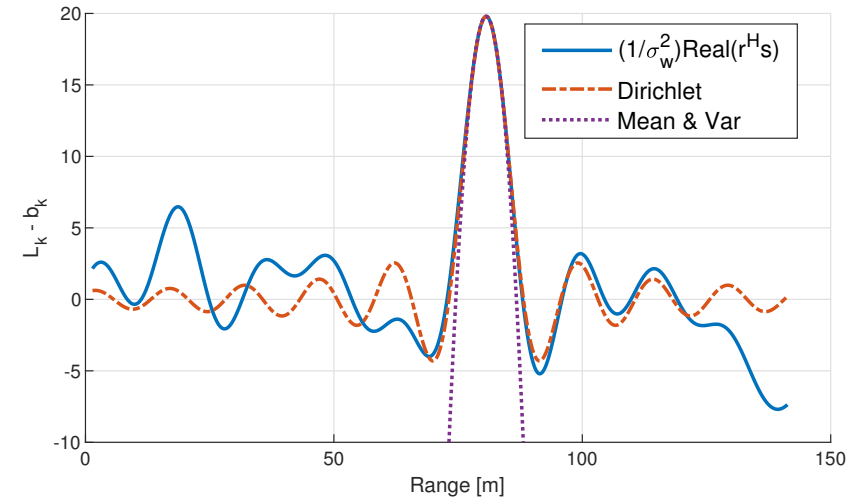

(a) Log-likelihood as defined in (9) omitting the constant term $b_{k}$

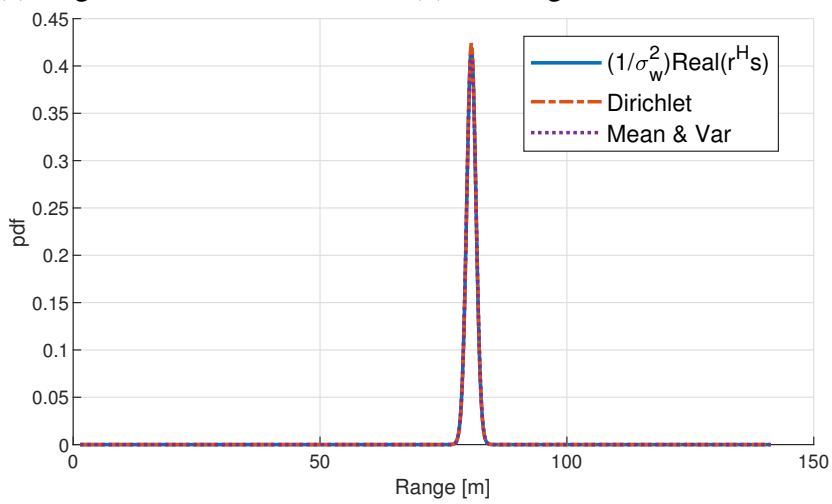

(b) Normalized range PDF

Fig. 3: Range log-likelihood and range distribution representation for $\mathrm{SNR}=-5 \mathrm{~dB}$

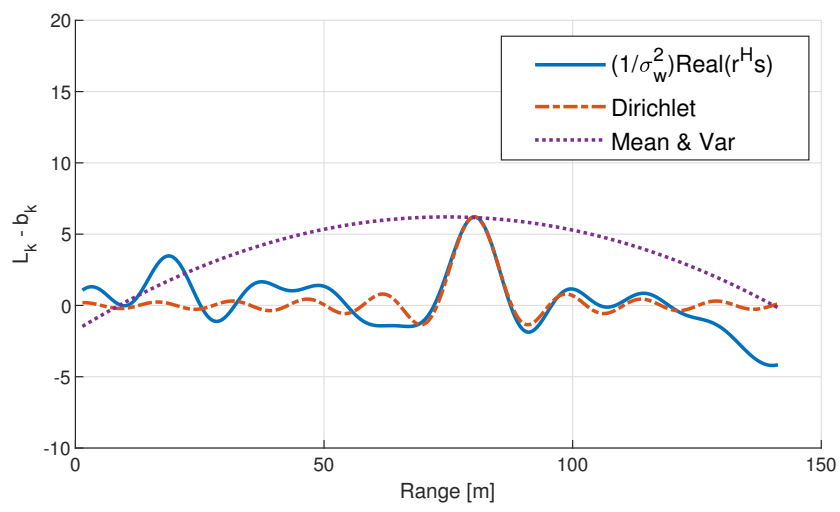

(a) Log-likelihood as defined in (9) omitting the constant term $b_{k}$

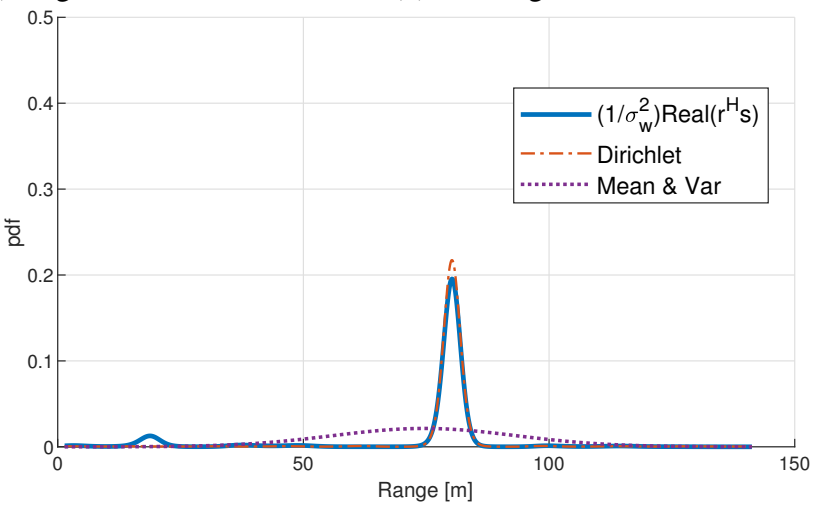

(b) Normalized range PDF

Fig. 4: Range log-likelihood and range distribution representation for $\mathrm{SNR}=-10 \mathrm{~dB}$
Similarly, Figures $3 b$ and $4 b$ show the range PDF for different SNR values ( -5 and $-10 \mathrm{~dB}$ respectively). On the one hand, for high SNR, both models represent the main lobe of the range log-likelihood well enough, so the normalized range distributions are practically overlapped (See Figure 3b). On the other hand, for lower SNR, using the range mean and variance as in [6] takes into account the side lobes generated by noise; thus, it distorts the shape of the multi-modal range PDF degrading the performance (See Figure $4 b$ ).

When the main lobe is comparable to the noise lobes, it is possible to select a wrong peak at iteration one, but such error is corrected in the following iterations by adding the information gathered by all other BS.

\section{CONCLUSION}

In this work, we proposed an improvement to the IPE algorithm presented in [6] to reduce the loss of information due to the processing with local information. The localization is done by means of an iterative process, in which each BS shares just a few parameters between all other BSs, hence the transmitter position is available at each BS at the end of each iteration. Numerical results show that the performance of the final algorithm gets close to the performance of a direct localization.

\section{ACKNOWLEDGMENT}

The authors acknowledge the financial support of the Walloon Region through the WIN2Wal/2018/1/DI/34 LUMINET project.s

\section{REFERENCES}

[1] "Evolved universal terrestrial radio access (e-utra); physical channels and modulation (release 13)," in Technical Specification 36.211 V13.13.0, January 2020.

[2] P. Closas, C. Fernandez-Prades, and J. A. Fernandez-Rubio, "Maximum likelihood estimation of position in gnss," IEEE Signal Processing Letters, vol. 14, no. 5, pp. 359-362, 2007.

[3] A. J. Weiss, "Direct position determination of narrowband radio frequency transmitters," IEEE Signal Processing Letters, vol. 11, no. 5, pp. 513-516, 2004.

[4] R. Zekavat and R. M. Buehrer, Source Localization: Algorithms and Analysis, 2019, pp. 59-106.

[5] A. Amar and A. J. Weiss, "New asymptotic results on two fundamental approaches to mobile terminal location," in 2008 3rd International Symposium on Communications, Control and Signal Processing, 2008, pp. $1320-1323$.

[6] F. Horlin, M. Van Eeckhaute, T. Van der Vorst, A. Bourdoux, F. Quitin, and P. De Doncker, "Iterative toa-based terminal positioning in emerging cellular systems," in 2017 IEEE International Conference on Communications (ICC), 2017, pp. 1-5.

[7] M. Van Eeckhaute, T. Van der Vorst, F. Quitin, P. De Doncker, and F. Horlin, "Toa-based iterative localization in rich multipath channels," in 2018 IEEE 29th Annual International Symposium on Personal, Indoor and Mobile Radio Communications (PIMRC), 2018, pp. 1-5.

[8] M. Van Eeckhaute, T. Van der Vorst, A. Bourdoux, F. Quitin, P. De Doncker, and F. Horlin, "Low complexity iterative localization of time-misaligned terminals in cellular networks," IEEE Transactions on Vehicular Technology, vol. 67, no. 11, pp. 10730-10739, 2018.

[9] S. Monfared, A. Delepaut, M. Van Eeckhaute, P. De Doncker, and F. Horlin, "Iterative localization method using aoa for iot sensor networks," in 2019 IEEE 89th Vehicular Technology Conference (VTC2019-Spring), 2019, pp. 1-6.

[10] M. Eeckhaute, E. Gottlob, P. Doncker, and F. Horlin, "Iterative rtof-based localization and time synchronization in wlan-like systems," Electronics Letters, vol. 54, 102018. 